Article

\title{
The Aborted Microspores (AMS)-Like Gene Is Required for Anther and Microspore Development in Pepper (Capsicum annuum L.)
}

\author{
Jinju Guo ${ }^{1,+}$, Chen Liu ${ }^{1,2,+}$, Peng Wang ${ }^{1}$, Qing Cheng ${ }^{1}$, Liang Sun ${ }^{1}$, Wencai Yang ${ }^{1}$ (1) and \\ Huolin Shen ${ }^{1, *}$ \\ 1 Beijing Key Laboratory of Growth and Developmental Regulation for Protected Vegetable Crops, \\ Department of Vegetable Science, College of Horticulture, China Agricultural University, Beijing 100193, \\ China; gjj1987.cool@163.com (J.G.); lcis250@163.com (C.L.); JLNWP2017@163.com (P.W.); \\ chengqing2013@126.com (Q.C.); liang_sun@cau.edu.cn (L.S.); yangwencai@cau.edu.cn (W.Y.) \\ 2 Shandong Key Laboratory of Greenhouse Vegetable Biology, Institute of Vegetables and Flowers, \\ Shandong Academy of Agricultural Sciences, Shandong Branch of National Vegetable Improvement Center, \\ Jinan 250100, China \\ * Correspondence: shl1606@cau.edu.cn \\ + These authors contributed equally to this work.
}

Received: 21 March 2018; Accepted: 30 April 2018; Published: 2 May 2018

\begin{abstract}
Pepper (Capsicum annuum L.) is an economically important vegetable crop worldwide. Although many genes associated with anther and pollen development have been identified, little is known about the mechanism of pollen abortion in pepper. Here, we identified and isolated two putative aborted microspore (AMS) isoforms from pepper flowers: CaAMS1 and CaAMS2. Sequence analysis showed that CaAMS2 was generated by retention of the fourth intron in CaAMS1 pre-mRNA. CaAMS1 encodes a putative protein with a basic helix-loop-helix (bHLH) domain belonging to the MYC subfamily of bHLH transcription factors, and it is localized to the nucleus. Truncated CaAMS2-1 and CaAMS2-2 are produced by alternative splicing. Quantitative real-time PCR analysis showed that CaAMS (referred to CaAMS1 and CaAMS2-2) was preferentially expressed in stamens and its expression level gradually decreases with flower development. RNA in situ hybridization analysis showed that CaAMS is strongly expressed in the tapetum at the tetrad and uninucleate stages. Downregulation of CaAMS led to partial shortened filaments, shriveled, indehiscent stamens and abortive pollens in pepper flowers. Several genes involved in pollen exine formation were downregulated in defective CaAMS-silenced anthers. Thus, CaAMS seems to play an important role in pepper tapetum and pollen development by regulating a complex genetic network.
\end{abstract}

Keywords: Capsicum annuum L.; CaAMS; bHLH transcription factor; MYC family; male sterility

\section{Introduction}

Anther and pollen development play a crucial role in the life cycle of flowering plants. The number of anther-specific transcripts and sterility-specific transcripts in plants indicate a complex biological process occurring between gametophytic and sporophytic tissues [1-5]. Anthers are surrounded by four distinct somatic layers: the epidermis, endothecium, middle layer, and tapetum, from surface to interior [6]. The tapetum is the innermost layer of the anther wall that surrounds the locule and directly contacts with microspores [6,7]. As a secretory layer, tapetal cells play a crucial role in microspore development by supplying metabolites, nutrients, and sporopollenin precursors [8]. Therefore, the development of tapetal cells differentiation, tapetum development, and subsequent degradation are all closely associated with pollen fertility. 
SPOROCYTELESS (SPL)/NOZZLE (NZZ) was one of the first genes identified in early anther cell division and differentiation in Arabidopsis thaliana. Because $s p l / n z z$ mutants show normal archesporial cells but defective cell division, these mutants produce aborted microspores and are male sterile [2,9]. SPL/NZZ regulates early anther cell differentiation by activating downstream genes [10]. Previous research on $A$. thaliana proposed that the leucine-rich repeat receptor-like kinases complex consisting of EXCESS MICROSPOROCYTES1 (EMS1)/EXTRA SPOROGENOUS CELLS (EXS), SOMATIC EMBRYOGENESIS RECEPTOR LIKE KINASE 1 and 2 (SERK1 and SERK2), and TAPETUM DETERMINANT 1 (TPD1) regulate cell-type specification and differentiation. The EMS1/EXS gene encodes a leucine-rich repeat receptor protein kinase which putatively localizes to the cell surface and likely plays an essential role in cell-to-cell communication [11,12]. The disruption of EMS1 leads to the absence of tapetal cells and to the production of sterile pollen grains. Additionally, serk1 serk2 double mutants are phenotypically similar to ems1/exs mutants [13,14]. TPD1 encodes a small secreted protein that possibly works in coordination with the EMS1/EXS gene product to determine tapetal cells fate in A. thaliana [15]. A recently proposed model suggested that TPD1 acts as ligand in its interaction with EMS1/EXS and SERK1/2 to specify tapetal cell fate $[4,16]$.

Other genes involved in late tapetum development or function in $A$. thaliana were also reported. These included DYSFUNCTIONAL TAPETUM 1 (DYT1), MALE STERILITY 1/2 (MS1/2), ABORTED MICROSPORE (AMS), and AtMYB103. DYT1 encodes a putative helix-loop-helix (bHLH) transcription factor and plays a crucial role in tapetal differentiation and early function [17]. The mRNA expression level of DYT1 in $s p l / n z z$ and ems1/exs mutants suggested that this gene might act on downstream SPL/NZZ and EMS1/EXS [17]. AMS also encodes a bHLH transcription factor and plays a crucial role in tapetum development and post-meiotic microspore development [18]. MS1 encodes a protein homologous to the plant homeodomain (PHD)-finger family of transcription factors. Its mutation results in premature degeneration of tapetal cells and complete male sterility [19]. The notably reduced expression levels of MS1 and AMS in dyt1 mutants suggested that both genes act on downstream DYT1 [17]. The protein encoded by MS2 shows high homology to a fatty acyl reductase that converts modified lauric acids to sporopollenin precursors, and $m s 2$ mutations produce nonviable pollen without exine layer [20]. MYB80 (MYB103), which is also required for tapetum and microspore development [21], works downstream of $A M S$ and only expresses in the tapetum of developing anthers [22]. The reduced transcript levels of $M S 1, M S 2, A 6$, and UNDEAD in myb80 mutants suggested these genes work on downstream MYB80 [23,24].

In the present study, we isolated two $A$. thaliana AMS-like isoforms from pepper (Capsicum annuum L.) flower buds, which were designated CaAMS1 and CaAMS2. Two truncated CaAMS2-1 and CaAMS2-2 were predicted for fourth intron retention in CaAMS2 precursor messenger RNA (pre-mRNA). As CaAMS is preferentially expressed in stamens, its expression level gradually decreased with the development of the flower buds. RNA in situ hybridization showed that CaAMS was strongly expressed in the tapetum at the tetrad and early-mid uninucleate stages. Downregulation of CaAMS results in partial shortened filaments, shriveled, indehiscent stamens, and abortive pollens in pepper flowers. Several genes involved in pollen exine formation were significantly downregulated in defective CaAMS-silenced anthers. Overall, these traits indicate that $C a A M S$ plays an important role in pepper tapetal and pollen development by means of a complex genetic network. Therefore, CaAMS seems to be a practical and effective tool to artificially regulate stamens' fertility and further improve the efficiency of breeding practices.

\section{Results}

\subsection{Isolation and Sequence Analysis of CaAMS Genes from Pepper}

We isolated two putative AMS isoforms from pepper flower buds, designated as CaAMS1 (Accession No. MH230199) and CaAMS2. Through sequence comparison, we found that CaAMS2 was generated by retention of the fourth intron (Accession No. MH230200) in CaAMS1 pre-mRNA. The coding sequence of CaAMS1 contained 1788 bp encoding 595 amino acids, and the predicted 
molecular weight and isoelectric point of this protein were $68.13 \mathrm{kDa}$ and 5.83 , respectively. The exon/intron structure prediction showed that CaAMS1 has eight exons and seven introns (Figure 1A). The retention-generated CaAMS2 was 2144 bp with a 356-bp intron insertion between exons 4 and 5 in CaAMS1. This alternative splicing probably resulted in a premature translational termination of CaAMS2 at $495 \mathrm{bp}$ (Figure 1A,B) or in an open reading frame (ORF) shift from $852 \mathrm{bp}$ (Figure 1A,C), as predicted using ORF finder. These two truncated CaAMS2s were called CaAMS2-1 (Figure 1B) and CaAMS2-2 (Figure 1C), respectively. The basic local alignment search tool (BLAST) analysis indicated that the deduced CaAMS1 was highly similar to the AMS homologs found in other species and contained a bHLH domain belonging to the MYC subfamily of bHLH genes (Figure 1B,C). The N-terminus truncated CaAMS2-2 retained the bHLH domain, while the C-terminus truncated CaAMS2-1 only retained the N-terminal region characteristic of MYB and MYC transcription factors. Thus, the subsequent analyses mainly focused on CaAMS1 and CaAMS2-2.

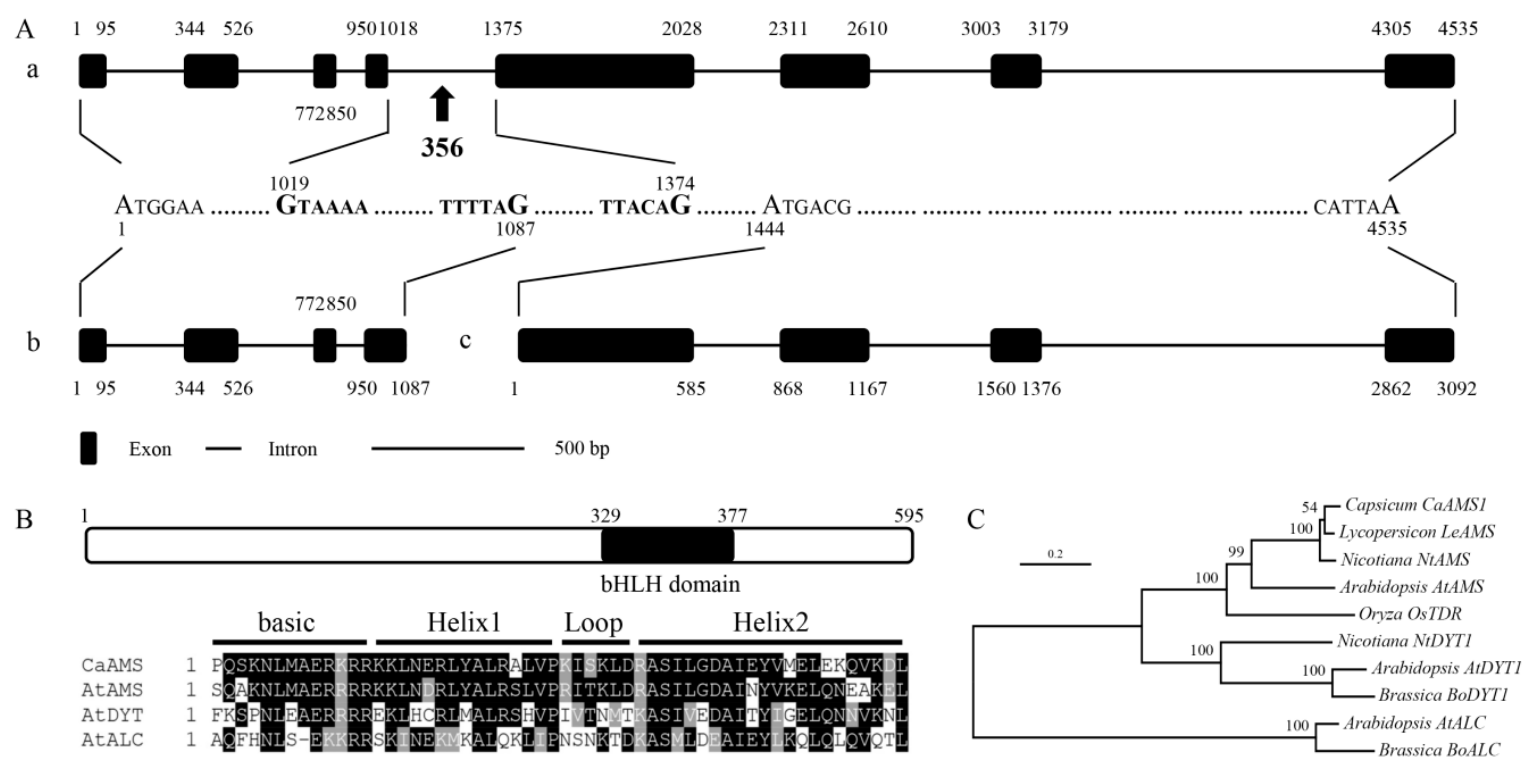

Figure 1. Sequence analysis of CaAMS. (A) Exon-intron structure of CaAMSs. a-c indicate the exon-intron structures of CaAMS1, CaAMS2-1, and CaAMS2-2, respectively. Black boxes indicate exons; Connecting lines indicate introns; Letters in bold indicate the fourth introns inserted; Capital letters indicate the starting and stopping nucleotides. (B) Predicted protein structure of CaAMS1 and sequence alignment of the bHLH domain. The black region indicates the bHLH domain. (C) Phylogenetic analysis of the predicted CaAMS1 proteins with other MYC class bHLH proteins. Amino accession numbers are listed as follows: (1) Arabidopsis thaliana: AtAMS, AT2G16910.1; AtDYT1, AT4G21330; AtALC, NP_201512; (2) Lycopersicum esculentum: LeAMS, XP_019070622; (3) Nicotiana tabacum: NtAMS, XP_016448229; NtDYT1, XP_009773859; (4) Brassica oleracea: BoDYT1, XP_013669215; BoALC, XP_013686240; (5) Oryza sativa: OsTDR, Q6YUS3. The bootsrap values from 1000 replicates were indicated on most major nodes.

\subsection{Expression Patterns of CaAMS Genes}

The expression patterns of CaAMS (except CaAMS2-1) in different whorls of floral buds at different developmental stages (i.e., the tetrad, early-mid uninucleate, late uninucleate, binucleate, and maturing stages) were analyzed using quantitative real-time qRT-PCR (Figure 2A). Semi-quantitative RT-PCR analysis was only used for anthers at different developmental stages (Figure 2B). Results showed that CaAMS was preferentially expressed in stamens and its expression level gradually decreased with the development of floral buds (Figure 2A,B). Results of RNA in situ hybridization further revealed CaAMS signaling cannot be detected in tapetum at pollen mother cell stage (Figure 2C). However, 
CaAMS was strongly expressed in the tapetum at the tetrad and uninucleate stages (Figure 2D-F). Therefore, CaAMS might play an important role in pepper tapetum and pollen development.
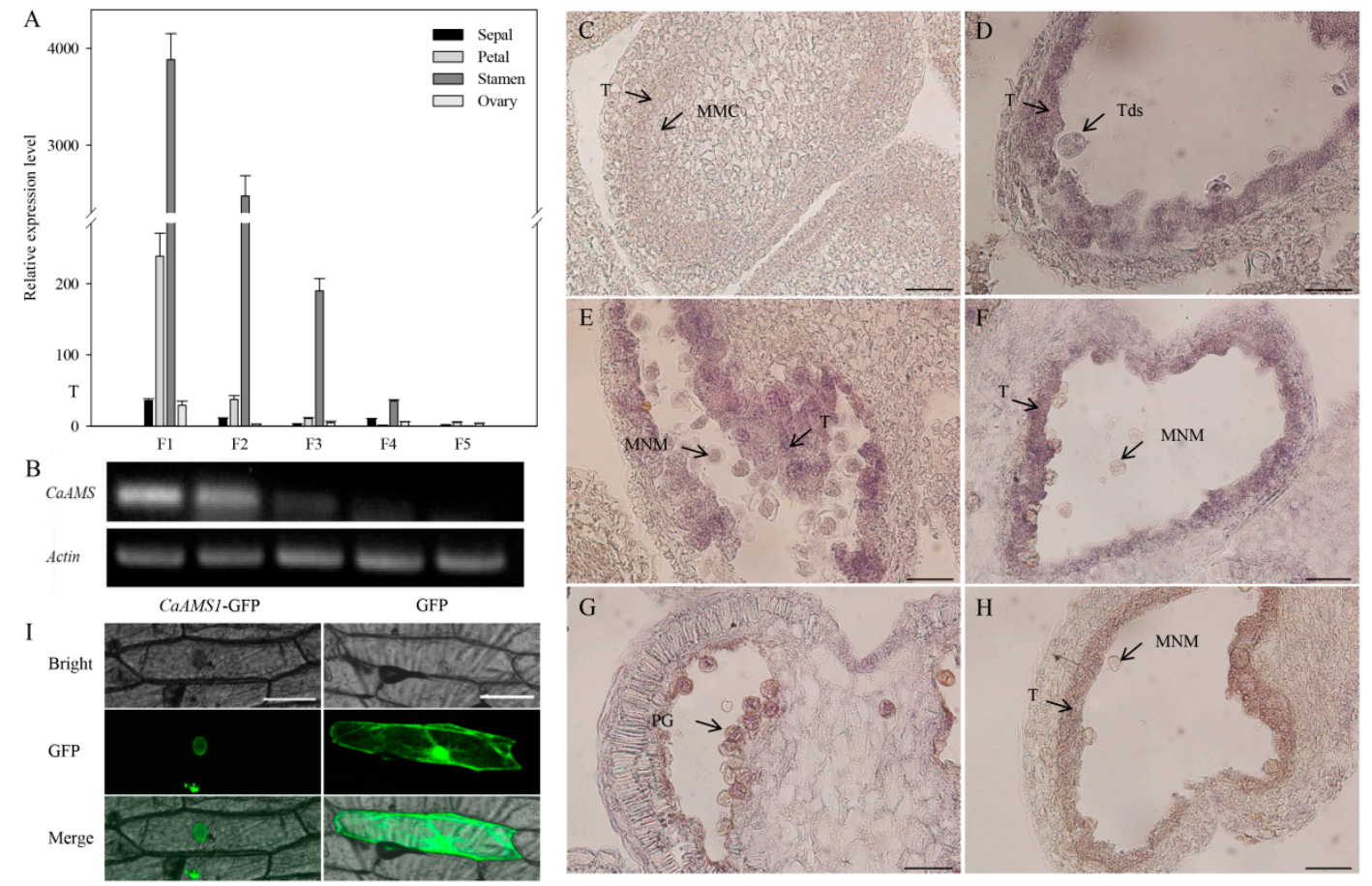

Figure 2. Expression analysis of CaAMS in flower buds and subcellular localization of CaAMS1 protein. (A) qRT-PCR analysis of CaAMS in flower buds at various developmental stages. F1, F2, F3, F4, and F5 indicate flower buds at tetrad, early-mid uninucleate, late uninucleate, binucleate, and maturing stages, respectively. Data are represented as means $\pm \mathrm{SD}(n=3)$. (B) Semi-quantitative RT-PCR analysis of CaAMS in stamens at various developmental stages (same as in qRT-PCR analysis). (C-H) RNA in situ hybridization with the CaAMS probe. (C): CaAMS signal cannot be detected in tapetum at pollen mother cell stage. (D-F) Strong signal is detected in the tapetum at the tetrad and uninucleate stages. (G) CaAMS signal cannot be detected at pollen mature stage. $(\mathbf{H})$ The negative control in the uninucleate stage. Only background signal can be detected. T indicates the tapetum; Td indicates the tetrad; MMc indicates the microspore mother cell. MNM indicates the uninucleate microspore. PG indicates the pollen grain. Bar $=200 \mu \mathrm{m}$. (I) Subcellular localization of CaAMS protein in onion epidermal cells. Bars $=100 \mu \mathrm{m}$.

\subsection{Subcellular Localization of CaAMS1}

After fusing the coding region of CaAMS1 to the N-terminus of green fluorescence protein (GFP) to produce a CaMV 35S-CaAMS-GFP fusion protein, we performed transient expression assays in epidermal cells of onion to detect the subcellular localization of CaAMS1. This result showed that CaAMS1 is a nuclear localized protein which matched well with the characteristic of transcription factors (Figure 2I).

\subsection{Virus-Induced Silencing of CaAMS Induces Partial Male Sterility}

Virus-induced gene silencing (VIGS) was evaluated by silencing the endogenous tomato PHYTOENE DESATURASE (PDS) gene on TRV2:PDS-treated plants. Twenty days after Agrobacterium tumefaciens-infiltration, tender leaves in about $80 \%(n=10)$ of the plants appeared photobleached (Figure 3A). Identification based on PCR results indicated that about $65 \%$ of the seedlings were successfully transformed. Photobleaching was also detected in flowers (Figure 3B). 
There was no obvious phenotype change in the vegetative growth stage. Defective flowers, appearing on the second layer of flowers $(40 \%, n=35)$, showed partial shortened filaments, shriveled, indehiscent stamens, and abortive pollens (Figure 3C,D). We designated these defective flowers as CaAMS-1, and normal flowers as CaAMS-0, in TRV2:CaAMS-treated flowers. Each defective flower showed one to three degenerated stamens (five stamens per flower) (Figure 3C). The qRT-PCR analysis showed notably lower transcript levels of CaAMS in CaAMS-1 flower stamens (Figure 3G). The expression levels of CaAMS in CaAMS-0 flower stamens also decreased, but not differ significantly from that of TRV2-treated flower stamens (Figure 3G). These results further indicated that CaAMS might play an essential role in regulating stamen and pollen development in pepper.
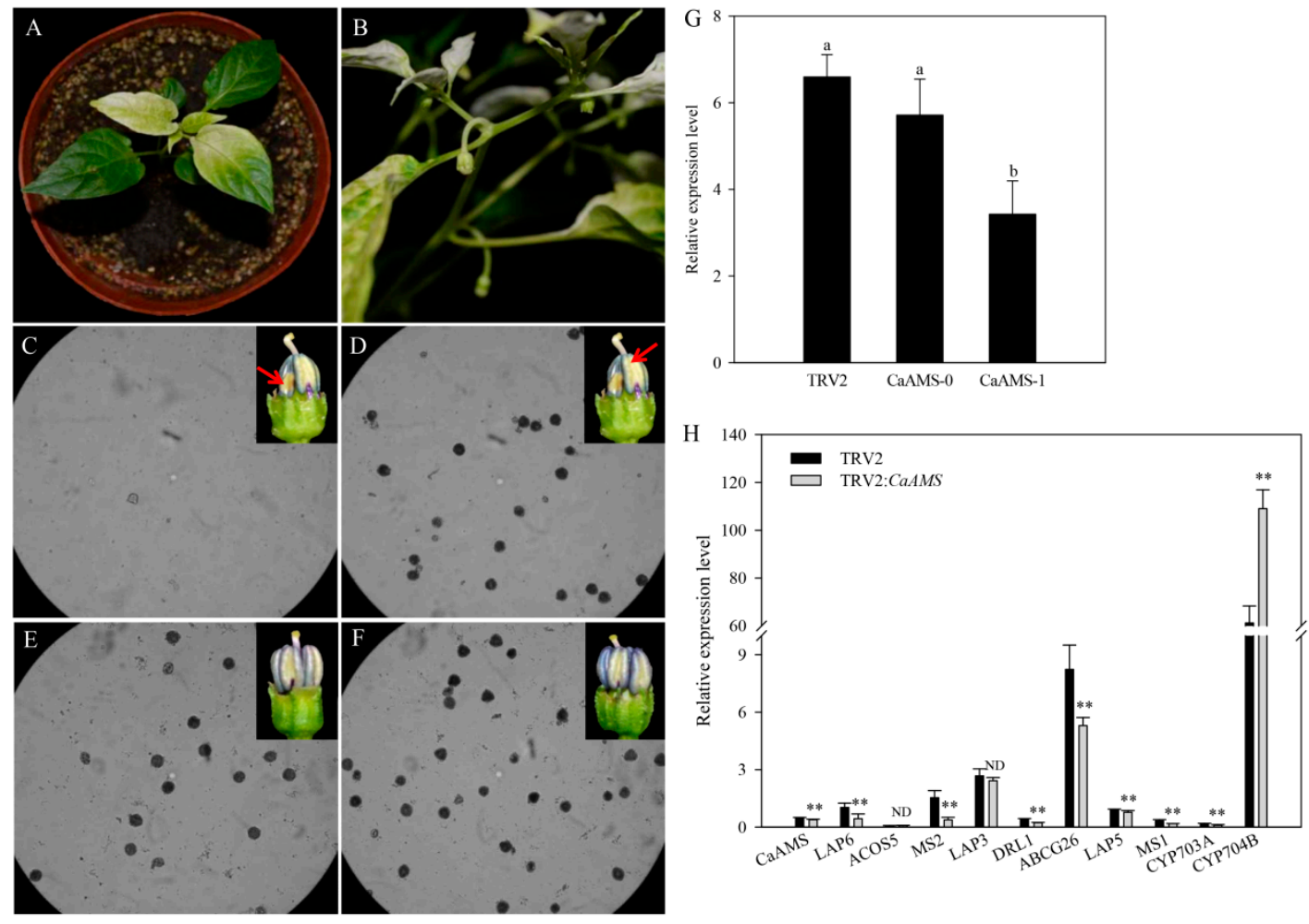

Figure 3. Effects of silencing CaAMS in pepper. (A,B) PDS-silenced plants (negative controls). (C-E) CaAMS-silenced plants. (C) Pollen grains in defective stamens of CaAMS-1 type flower buds. (D) Pollen grains in normal stamens of CaAMS-1 type flower buds. (E) Pollen grains in stamens of CaAMS-0 type flower buds. (F) Pollen grains in TRV2-treated stamens. (G) qRT-PCR analysis of CaAMS in CaAMS-silenced flowers with various phenotypes. Different letters above bars indicate significant differences according to Duncan's multiple range test $(p<0.05)$. (H) Relative expression analysis of genes involved in pollen exine formation by qRT-PCR. CYP703A, Cytochrome P450 703A; CYP704B, Cytochrome P450 704B; LAP3/5/6, Less adhesive pollen 3/5/6; MS1/2, Male sterility 1/2; DRL1, Dihydroflavonol 4-reductase-like 1; ABCG26, ATP-binding cassette transporter G26; ACOS5, Acetyl-CoA synthetase 5. ${ }^{* *}$ indicates significant differences at $p<0.01$, respectively. ND means no difference. Data are represented as means $\pm \mathrm{SD}(n=3)$.

\subsection{Genes Involved in Pollen Formation Have Altered Expression in CaAMS-Silenced Anthers}

We performed qRT-PCR using RNA from CaAMS-silenced anthers to evaluate if the downregulation of $C a A M S$ would affect other genes related to pollen development. Primers for CaAMS and MS1 are listed in Supplementary Table S1 and those for other genes were designed based on our previous work [25]. The expression level of LAP5/6, MS1/2, DRL1, ABCG26 and CYP703A were considerably reduced in $C a A M S$-silenced anthers. This indicated that these genes might work downstream CaAMS and that their normal expression might depend on CaAMS. The expression level 
of $A C O S 5$ and $L A P 3$ were slightly altered, and the transcript level of CYP704B was notably increased in CaAMS silenced anthers, indicating that their expression does not rely on the CaAMS gene (Figure $3 \mathrm{H}$ ).

\subsection{Promoter Analysis}

A 1997-bp DNA fragment upstream the CaAMS start codon was cloned and regarded as the CaAMS promoter (Accession No. MH230200). To investigate the regulation mechanisms of CaAMS, we analyzed the regulatory elements in the CaAMS promoter region using the PlantCARE database. Twenty-six cis-elements associated with light responsiveness, two ABRE elements involved in abscisic acid (ABA) responsiveness, two methyl jasmonate (MeJA)-responsive motifs (CGTCA/TGACG-motif), one salicylic acid (SA) responsive element (TCA-element) and gibberellin (GA3)-responsive element (GARE-motif) were identified (Figure 4A). Several cis-acting elements involved in defense and stress (e.g., heat, drought) responsiveness were also identified (Supplementary Table S2). The uneven distribution of cis-elements in the CaAMS promoter suggested that its transcription is regulated by various environmental signals such as light, hormones, or stress. Promoter deletion analysis indicated that the core region of CaAMS is localized at -518 to -1056 bp upstream the ATG translation initiation codon. Inhibitory factors might localize from -1 to $-518 \mathrm{bp}$ (Figure $4 \mathrm{~B}$ ).

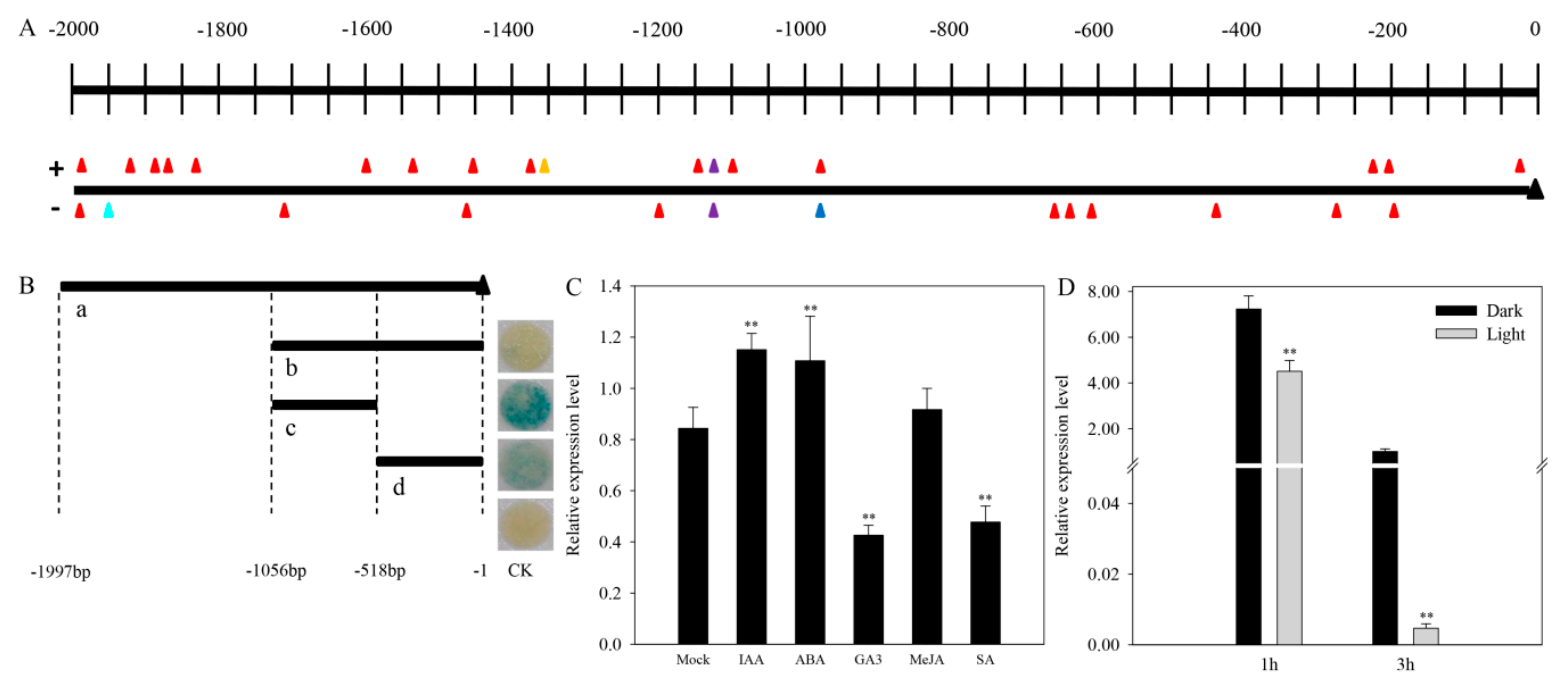

Figure 4. CaAMS promoter analysis and expression patterns of CaAMS in response to exogenous hormones and light. (A) Predicted cis-acting elements of the CaSEP5 promoter. Red represents light responsive elements; Blue indicates ABA responsive elements; Purple indicates MeJA responsive elements; Orange indicates SA responsive elements; Aqua indicates GA responsive elements. (B) Deletion analysis of the CaAMS promoter. Label a indicates the CaAMS promoter, labels $\mathbf{b}-\mathbf{d}$ indicate four truncated promoter fragments and their corresponding GUS expression in tobacco leaf disks. (C) Expression patterns of CaAMS in response to exogenous hormones. (D) Expression patterns of CaAMS in response to light. ${ }^{* *}$ indicates significant differences at $p<0.01$, respectively. ND means no difference. Data are represented as means $\pm \operatorname{SD}(n=3)$.

\subsection{CaAMS Response to Hormones and Light}

Cis-element analysis suggested that transcript levels of CaAMS might be regulated by various environmental signals, including hormones and light. The expression of $C a A M S$ was notably reduced under $100 \mu \mathrm{mol} \mathrm{GA3}$ and $100 \mu \mathrm{mol} \mathrm{SA}$ treatments, but greatly increased under $100 \mu \mathrm{mol}$ indole acetic acid (IAA) and $100 \mu \mathrm{mol}$ ABA treatments; however, CaAMS transcript levels showed no response to $100 \mu \mathrm{mol}$ MeJA (Figure 4C). Light treatment considerably reduced CaAMS expression (Figure 4D). These results further illustrated that CaAMS transcript levels might be regulated by hormones and light. 


\section{Discussion}

\subsection{Structure and Localization of the CaAMS Gene}

MYC class transcription factors are reported to play key roles in cell proliferation, differentiation, and apoptosis [26]. The MYC family proteins consist of three distinct family members, c-MYC, L-MYC, and N-MYC, arising from gene duplication during early evolution [27]. Despite their differences, MYC family members are assumed to work through similar mechanisms [28] and proposed to form homodimers or heterodimers with MYC-associated factor X (MAX) proteins via their helix-loop-helix (HLH) domain $[29,30]$. The MYC/MAX heterodimers bind variants of the E-box motif "CANNTG", which can be found in promoters or transcribed sequences of MYC target genes and such binding usually activates the target gene [31,32].

Basic helix-loop-helix proteins are widely distributed in eukaryotic kingdoms. They constitute the largest families of transcription factors and control many biological processes [33,34]. Several MYC class bHLH proteins have been functionally characterized in plants. For example, delila (del) in Antirrhinum sp. regulates the pattern of red anthocyanin pigmentation [35], alcatraz (ALC) gene in Arabidopsis enabling cell separation in fruit dehiscence [36] and AMS in A. thaliana is involved in the development of tapetal cells and microspores, as well as in filament elongation [18].

In the present study, we isolated and characterized an $A$. thaliana AMS homolog transcription factor from pepper flower buds, named CaAMS, which encodes three predicted isoforms generated by alternative splicing. Alternative splicing of pre-mRNA is a regulated process during gene expression that results in multiple proteins encoded by a single gene. Almost all instances of alternative splicing involve one or more of the following basic modules: alternative 5' or 3' splice-site choice, cassette-exon inclusion or skipping, and intron retention [37,38]. While CaAMS1 contains the full-length pre-mRNA, CaAMS2 retains the fourth intron between exons 4 and 5 (Figure 1A). Intron-containing RNAs are frequently reported to retain in the nucleus, target for degradation, or repress translationally [39]. The CaAMS2 splice variant was predicted to encode two truncated proteins, designated CaAMS2-1 and CaAMS2-2 (Figure 1B,C). Conserved domain prediction indicated that CaAMS1 contained both the N-terminal region of MYC transcription factors and a bHLH domain, while CaAMS2-1 encoded a protein that only retained the N-terminal region and CaAMS2-1 a protein that only retained the bHLH domain. This domain comprises $~ 60$ conserved amino acids and two different functional sub-domains: a DNA binding basic region and two amphipathic $\alpha$-helices separated by a diverged loop region (i.e., HLH). The N-terminal basic region is involved in DNA binding through the E-box DNA motif "CANNTG", and the HLH domain promotes the formation of homodimeric or heterodimeric complexes $[40,41]$.

\subsection{CaAMS1 Is Required for Tapetum and Microspore Development in Pepper}

In flowering plants, male sterility depends on the normal development of anthers and microspores. The tapetum is the inner most layer of the anther wall and directly contacts with gametophytes. It is considered to play an essential role in the development of microspores to pollen grains, by supplying nutrients, metabolites, and sporopollenin precursors [42]. Manipulation of crop fertility has significant commercial value for F1 hybrid seed production. Although male-sterile lines have been widely used in pepper breeding, little is known on the mechanism of pollen abortion. Several male-sterile mutants have been associated with tapetum defects [43].

Although the precise mechanisms underlying tapetal development remain highly elusive, several genes involved in tapetum formation, development, and programmed cell death (PCD) have been identified and characterized [44]. In A. thaliana, the AMS gene encoding a bHLH protein plays a crucial role in the differentiation of tapetal cells and microspores within the developing anther [18]. The predicted CaAMS1 protein showed the highest similarity to AMS homologues in Solanum lycopersicum, and only $45 \%$ similarity to A. thaliana AMS, although all AMS homologues 
contain a bHLH domain belonging to the MYC class of bHLH transcription factors. Nevertheless, CaAMS1 shares similar exon/intron structures with A. thaliana AMS with eight exons and seven introns.

The qRT-PCR analysis indicated that $C a A M S$ is preferentially expressed in the stamens of flower buds at the tetrad stage, and that its transcription level gradually decreased as flower buds developed (Figure 2A,B). The RNA in situ hybridization further revealed that CaAMS was strongly expressed in the tapetum at the tetrad and the uninucleate stages (Figure 2D-F). However, CaAMS is not specific to the tapetum like AMS in Arabidopsis or TDR in rice [5,18]. The CaAMS signal was also detected in sepals, petals, and ovaries (Figure 2A). Therefore, the intron-containing alternative splicing might have affected the expression pattern of CaAMS in pepper.

Because pepper is highly recalcitrant to in vitro regeneration and genetic transformation, the function of CaAMS was evaluated using VIGS. No obvious changes were detected in the phenotype of the vegetative growth stage, but defective flowers appeared on the second layer of flowers. As VIGS is a transient silencing system that cannot completely silent the target genes, the altered phenotypes in CaAMS-silenced flowers were usually unstable. Only $40 \%$ of the flowers $(n=35)$ were defective, with shortened filaments, shriveled stamens, and abortive pollens. This is consistent with the phenotype of ams mutants in A. thaliana [18]. Additionally, not all (five) but only one to three stamens degenerated in each defective CaAMS-silenced flower. The qRT-PCR analysis showed that CaAMS transcript levels were considerably lower in CaAMS-1 type flower stamens than in normal flower stamens. However, in CaAMS-0 type flower stamens, the transcript levels of CaAMS were not considerably reduced in relation to control/normal flower stamens (Figure 3G). These results indicate that $C a A M S$ might play an essential role in regulating stamen and pollen development in pepper.

Previous studies suggested that AMS works downstream the TAPETAL DEVELOPMENT AND FUNCTION 1 (TDF1) gene encoding a putative R2R3MYB transcription factor, and that it plays a key role in tapetal differentiation and function [22]. Several tapetum-preferential genes have been identified as involved in the network regulating tapetal cells and microspores development. The AMS gene was reported to indirectly induce tapetal PCD or to regulate the downstream pathway of tapetal and pollen formation [44,45]. To test if CaAMS downregulation could affect putative pollen formation genes, we performed qRT-PCR using RNA from stamens of defective CaAMS-silenced flowers and 10 putative genes involved in pollen exine formation, based on our previous work [25]. Sporopollenin is one of the main components of pollen exine. During the sporopollenin precursor synthetic process, acetyl-CoA released from mitochondria was used as a substrate during fatty acid synthesis (FAS) formation in plastids. After C12, C16 and C18 fatty acids were synthesized, they were modified by Acyl-CoA synthetase5 (ACOS5) and then were transferred to the endoplasmic reticulum (ER). After hydroxylation by CYP703A and CYP704B, the products are CoA-esterified again by ACOS5. Finally, the products were converted to sporopollenin precursors by downstream MS2 and LAP5/ 6 [25,46-48]. ABCG26 was considered to be involved in tapetum-to-microspore sporopollenin monomer transport in Arabidopsis [49]. DRL1 and LAP3 were required to synthetic the flavonoids which may serve as sporopollenin precursors [50,51]. Any defects in this process would cause exine formation defect and pollen abortion. In this study, we found that the expression levels of $L A P 5 / 6$, $M S 1 / 2, D R L 1, A B C G 26$, and CYP703A were considerably reduced in defective CaAMS-silenced anthers (Figure $3 \mathrm{H}$ ), suggesting that the normal expression of these genes might depend on the CaAMS gene. Similar mutant phenotypes, mRNA expression levels, and the characteristics of ACOS5, MS2, and CYP703A suggested these might act in a common biochemical pathway [46].

\section{Materials and Methods}

\subsection{Plant Materials and Growth Conditions}

A self-bred pepper (C. annuиm) line was used in this study. Plants designated for gene cloning, qRT-PCR, and RNA in situ hybridization were grown in experimental fields at China Agricultural University, Beijing, China. For gene cloning and qRT-PCR, flower buds at different development stages 
(tetrad, early-mid uninucleate, late uninucleate, binucleate, and maturing stages) and dissected floral organs (sepals, petals, stamens, and ovaries) were collected, immediately frozen in liquid nitrogen and stored at $-80^{\circ} \mathrm{C}$ until use. For VIGS, plant seedlings were grown under $22^{\circ} \mathrm{C} / 16 \mathrm{~h}$ day and $18{ }^{\circ} \mathrm{C} / 8 \mathrm{~h}$ night conditions in a phytotron.

\subsection{RNA Extraction and $q R T-P C R$ Analyses}

Total RNA was isolated from flower buds using the SV Total RNA Isolation System Kit (Promega Corp., Madison, WI, USA) according to the manufacturer's instructions. $1 \mu \mathrm{g}$ total RNA was used to synthesize the first-strand cDNA. Reverse transcription was performed with a PrimeScript ${ }^{\text {TM }}$ RT Kit (TaKaRa Bio Inc., Kusatsu, Shiga, Japan). After diluting cDNA products five times, $2 \mu \mathrm{L}$ aliquots were used for gene cloning and qRT-PCR analysis. The primers used are listed in Supplementary Table S1.

For gene cloning, specific primers were designed based on the pepper genome database (available online: http://peppersequence.genomics.cn/page/species/index.jsp). The amplified cDNA fragments were cloned into pMD 19-T vectors (TaKaRa Bio Inc.), and positive clones were then sequenced at Huada Genetic Sequence Company (Beijing, China).

The qRT-PCR was performed using a GoTaq ${ }^{\circledR}$ qPCR Master Mix (Promega) following the manufacturer's protocol on an ABI 7500 real-time PCR system (Applied Biosystems, Foster City, CA, USA), under $95{ }^{\circ} \mathrm{C}$ for $1 \mathrm{~min}$, followed by 40 cycles of $95{ }^{\circ} \mathrm{C}$ for $30 \mathrm{~s}$ and $60{ }^{\circ} \mathrm{C}$ for $1 \mathrm{~min}$. Actin (GQ337966.1) was used as the internal control for its stable expression level in different plant tissues and under variable hormones and abiotic stresses treatments [52]. The qRT-PCR was run with three biological replicates and three technical replicates. Analyses were performed as describes before [25]. The relative expression levels of the target genes were calculated using the $2^{-\Delta \Delta C t}$ method. The semi-quantitative RT-PCR was performed using the same CaAMS primers as the qRT-PCR. Cycling conditions were $3 \mathrm{~min}$ at $94{ }^{\circ} \mathrm{C}$ followed by 30 cycles of $30 \mathrm{~s}$ at $94{ }^{\circ} \mathrm{C}, 30 \mathrm{~s}$ at $54{ }^{\circ} \mathrm{C}$, and $40 \mathrm{~s}$ at $72{ }^{\circ} \mathrm{C}$.

\subsection{Sequence Analysis}

Open reading frames were predicted using ORF Finder. The physicochemical characteristics of the proteins were predicted with the ProtParam tool in ExPASy (available online: http:/ /web.expasy. $\mathrm{org} /$ protparam/). Deduced amino acid sequences were aligned using Clustal X v.2.0 (available online: http:/ / macdownload.informer.com/clustalx/versions/) and displayed with the BoxShade server v.3.21 (available online: https:/ / embnet.vital-it.ch/software/BOX_form.html). A neighbor-joining (NJ) phylogenetic tree was constructed in MEGA v.5.05 (available online: http:/ / macdownload.informer. $\mathrm{com} /$ mega-5/) with 1000 bootstrap replicates.

\subsection{Promoter Analysis}

The 1996-bp fragment upstream the CaAMS transcription initiation codon was amplified as the CaAMS promoter using specifically-designed primers based on the pepper genome database (available online: http:/ / peppersequence.genomics.cn/page/species/index.jsp). We analyzed the cis-elements in the CaAMS promoter using PlantCARE database (available online: http://bioinformatics.psb. ugent.be/webtools/plantcare/html/). Serial 5'- and 3'-deletion fragments (A-C) of the full-length promoter were amplified and inserted into the PCAMBIA 1391 vector (without 355 promoter) before the $\beta$-glucuronidase (GUS) reporter. The recombined vectors were transformed into the A. tumefaciens GV1301 line and then transformed into young tobacco (Nicotiana benthamiana) leaves by $A$. tumefaciens-mediated vacuum infiltration. A GUS-staining solution was used as described by Wang et al. [49]. The primer sequences used are listed in Supplementary Table S1.

\subsection{Subcellular Localization}

Full-length CaAMS genes (without the terminator codon) were inserted into PUC-SPYNE vectors digested with $\mathrm{XbaI}$ and $\mathrm{BamH1}$. Gold particles coated with recombinant plasmids were bombarded onto onion bulb epidermis using a Bio-Rad PDS-1000/He particle delivery system (Bio-Rad 
Laboratories Ltd., Hercules, CA, USA). The epidermises were cultured in Murashige-Skoog medium for $24 \mathrm{~h}$ at $25^{\circ} \mathrm{C}$, in dark. The GFP fluorescence was observed under an Olympus 1X71 confocal microscope (Olympus Corp., Shinjuku, Tokyo, Japan) at $488 \mathrm{~nm}$.

\subsection{Hormone and Light Treatments}

Pepper seedlings at full-blooming stage (i.e., starting to develop the forth layer flower buds) and with uniform growth were selected for treatments. For the exogenous hormone treatments, 10 flower buds at the tetrad stage were randomly collected and embed into gauze soaked with $100 \mu \mathrm{M}$ hormone solution (IAA, ABA, MeJA, SA, or GA3) in plastic petri dishes for $1 \mathrm{~h}$. For light treatments, five plants were covered with a black cloth for $1 \mathrm{~h}$ and $3 \mathrm{~h}$, and five plants were kept under normal lighting conditions (control group). Ten flower buds at the tetrad stage were randomly collected from the five treated plants and immediately frozen in liquid nitrogen. All the experiments were performed in triplicate.

\subsection{RNA In Situ Hybridization}

Fresh shoot apices and flower buds at different developmental stages were collected and fixed in formaldehyde-acetic acid alcohol, dehydrated in graded ethanol series, dewaxed in Histoclear (National Diagnostics, Atlanta, GA, USA), embedded in Paraplast, and sectioned into $10 \mu \mathrm{m}$ slices. Gene specific probes were generated by RNA polymerase using the DIG RNA labeling kit (Roche, Basel, Switzerland). RNA hybridization and hybridization signaling detection were performed according to Kouchi and Hata [53]. Primers are listed in Supplementary Table S1.

\subsection{VIGS}

The 329-bp C-terminal specific region of CaAMS was amplified and inserted into the vector pTRV2 at the BamH1 and Xhol1 sites. The recombined TRV2:CaAMS vector was transformed into A. tumefaciens GV1301. These transformants and pTRV1 were co-transformed into pepper seedlings with 1-2 euphyllae (three week-old-plants) by $A$. tumefaciens-mediated vacuum infiltration. The TRV2:PDS transformants were used as positive controls. Fifteen days after $A$. tumefaciens-infiltration, total RNA was extracted from young leaves to determine the infection efficiency of recombinant TRV in pepper plants. Successfully transformed plants were used for further analysis.

\section{Conclusions}

In conclusion, CaAMS is preferentially expressed in the tapetum at the tetrad and the early-mid uninucleate stages. Downregulation of CaAMS results in partial shortened filaments, shriveled, indehiscent stamens, and abortive pollens in pepper flowers. Several genes involved in pollen exine formation were downregulated in defective $C a A M S$-silenced anthers. These results indicate that CaAMS plays an important role in pepper tapetum and pollen development by regulating a complex genetic network.

Supplementary Materials: The following are available online at http:/ / www.mdpi.com/1422-0067/19/5/1341/ s1. Table S1. Primer sequences used in this study. Table S2. Cis-elements in the promoter region of CaAMS.

Author Contributions: J.G. performed most of the experiments, analyzed the data, and drafted the manuscript. C.L. performed the VIGS. P.W. performed hormone and light treatments. Q.C. performed the qRT-PCR analysis. W.Y. and L.S. revised the manuscript. H.S. designed and directed the entire study. All authors have read and approved the final manuscript.

Funding: This work was supported by the National Key Research and Development Program of China (2017YFD0101903) and the Beijing Fruit Vegetables Innovation Team of Modern Agricultural Industry Technology System (BAIC01-2018).

Conflicts of Interest: The authors declare no conflict of interest. 


$\begin{array}{ll}\text { Abbreviations } & \\ \text { bHLH } & \begin{array}{l}\text { basic helix-loop-helix } \\ \text { plant homeodomain }\end{array} \\ \text { PHD } & \begin{array}{l}\text { precursor messenger RNA } \\ \text { ppen reading frame }\end{array} \\ \text { ORF } & \text { basic local alignment search tool } \\ \text { BLAST } & \text { quantitative real-time PCR } \\ \text { qRT-PCR } & \text { virus-induced gene silencing } \\ \text { VIGS } & \text { abscisic acid } \\ \text { ABA } & \text { methyl jasmonate } \\ \text { MeJA } & \text { salicylic acid } \\ \text { SA } & \text { gibberellin } \\ \text { GA3 } & \text { indole acetic acid } \\ \text { IAA } & \text { myelocytomatosis } \\ \text { MYC } & \text { MYC-associated factor X } \\ \text { MAX } & \text { helix-loop-helix } \\ \text { HLH } & \text { programmed cell death } \\ \text { PCD } & \beta \text {-glucuronidase } \\ \text { GUS } & \end{array}$

\section{References}

1. Yang, W.C.; Ye, D.; Xu, J.; Sundaresan, V. The SPOROCYTELESS gene of Arabidopsis is required for initiation of sporogenesis and encodes a novel nuclear protein. Genes Dev. 1999, 13, 2108-2117. [CrossRef] [PubMed]

2. Wijeratne, A.J.; Zhang, W.; Sun, Y.; Liu, W.; Albert, R.; Zheng, Z.; Oppenheimer, D.G.; Zhao, D.; Ma, H. Differential gene expression in Arabidopsis wild-type and mutant anthers: Insights into anther cell differentiation and regulatory networks. Plant J. 2007, 52, 14-29. [CrossRef] [PubMed]

3. Ma, X.; Feng, B.M.; Ma, H. AMS-dependent and independent regulation of anther transcriptome and comparison with those affected by other Arabidopsis anther genes. BMC Plant Biol. 2012, 12, 23. [CrossRef] [PubMed]

4. $\mathrm{Ma}, \mathrm{H}$. Molecular genetic analyses of microsporogenesis and microgametogenesis in flowering plants. Annu. Rev. Plant Biol. 2005, 56, 393-434. [CrossRef] [PubMed]

5. Li, N.; Zhang, D.S.; Liu, H.S.; Yin, C.S.; Li, X.X.; Liang, W.Q.; Yuan, Z.; Xu, B.; Chu, H.W.; Wang, J.; et al. The rice Tapetum Degeneration Retardation gene is required for tapetum degradation and anther development. Plant Cell 2006, 18, 2999-3014. [CrossRef] [PubMed]

6. Goldberg, R.B.; Beals, T.P.; Sanders, P.M. Anther development: Basic principles and practical applications. Plant Cell 1993, 5, 1217-1229. [CrossRef] [PubMed]

7. Pacini, E.; Franchi, G.G.; Hesse, M. The tapetum: Its form, function, and possible phylogeny in Embryophyta. Plant Syst. Evol. 1985, 149, 155-185. [CrossRef]

8. Piffanelli, P.; Ross, J.H.E.; Murphy, D.J. Biogenesis and function of the lipidic structures of pollen grains. Sex. Plant Reprod. 1998, 11, 65-80. [CrossRef]

9. Schiefthaler, U.; Balasubramanian, S.; Sieber, P.; Chevalier, D.; Wisman, E.; Schneitz, K. Molecular analysis of NOZZLE, a gene involved in pattern formation and early sporogenesis during sex organ development in Arabidopsis thaliana. Proc. Natl. Acad. Sci. USA 1999, 96, 11664-11669. [CrossRef] [PubMed]

10. Balasubramanian, S.; Schneitz, K. NOZZLE regulates proximal-distal pattern formation, cell proliferation and early sporogenesis during ovule development in Arabidopsis thaliana. Development 2000, 127, 4227-4238. [PubMed]

11. Canales, C.; Bhatt, A.M.; Scott, R.; Dickinson, H. EXS, a putative LRR receptor kinase, regulates male germline cell number and tapetal identity and promotes seed development in Arabidopsis. Curr. Biol. 2002, 12, 1718-1727. [CrossRef]

12. Zhao, D.Z.; Wang, G.F.; Speal, B.; Ma, H. The EXCESS MICROSPOROCYTES1 gene encodes a putative leucine-rich repeat receptor protein kinase that controls somatic and reproductive cell fates in the Arabidopsis anther. Genes Dev. 2002, 16, 2021-2031. [CrossRef] [PubMed] 
13. Albrecht, C.; Russinova, E.; Hecht, V.; Baaijens, E.; de Vries, S. The Arabidopsis thaliana SOMATIC EMBRYOGENESIS RECEPTOR-LIKE KINASES1 and 2 control male sporogenesis. Plant Cell 2005, 17, 3337-3349. [CrossRef] [PubMed]

14. Colcombet, J.; Boisson-Dernier, A.; Ros-Palau, R.; Vera, C.E.; Schroeder, J.I. Arabidopsis SOMATIC EMBRYOGENESIS RECEPTOR KINASES1 and 2 are essential for tapetum development and microspore maturation. Plant Cell 2005, 17, 3350-3361. [CrossRef] [PubMed]

15. Yang, S.L.; Xie, L.F.; Mao, H.Z.; Puah, C.S.; Yang, W.C.; Jiang, L.X.; Sundaresan, V.; Ye, D. TAPETUM DETERMINANT1 is required for cell specialization in the Arabidopsis anther. Plant Cell 2003, 15, 2792-2804. [CrossRef] [PubMed]

16. Zhang, D.B.; Yang, L. Specification of tapetum and microsporocyte cells within the anther. Curr. Opin. Plant Biol. 2014, 17, 49-55. [CrossRef] [PubMed]

17. Zhang, W.; Sun, Y.J.; Timofejeva, L.; Chen, C.B.; Grossniklaus, U.; Ma, H. Regulation of Arabidopsis tapetum development and function by DYSFUNCTIONAL TAPETUM1 (DYT1) encoding a putative bHLH transcription factor. Development 2006, 133, 3085-3095. [CrossRef] [PubMed]

18. Sorensen, A.M.; Kröber, S.; Unte, U.S.; Huijser, P.; Dekker, K.; Saedler, H. The Arabidopsis ABORTED MICROSPORES (AMS) gene encodes a MYC class transcription factor. Plant J. 2003, 33, 413-423. [CrossRef] [PubMed]

19. Wilson, Z.A.; Morroll, S.M.; Dawson, J.; Swarup, R.; Tighe, P.J. The Arabidopsis MALE STERILITY1 (MS1) gene is a transcriptional regulator of male gametogenesis, with homology to the PHD-finger family of transcription factors. Plant J. 2001, 28, 27-39. [CrossRef] [PubMed]

20. Ariizumi, T.; Toriyama, K. Genetic regulation of sporopollenin synthesis and pollen exine development. Annu. Rev. Plant Biol. 2011, 62, 437-460. [CrossRef] [PubMed]

21. Higginson, T.; Li, S.F.; Parish, R.W. AtMYB103 regulates tapetum and trichome development in Arabidopsis thaliana. Plant J. 2003, 35, 177-192. [CrossRef] [PubMed]

22. Zhu, J.; Chen, H.; Li, H.; Gao, J.F.; Jiang, H.; Wang, C.; Guan, Y.F.; Yang, Z.N. Defective in Tapetal development and function 1 is essential for anther development and tapetal function for microspore maturation in Arabidopsis. Plant J. 2008, 55, 266-277. [CrossRef] [PubMed]

23. Zhang, Z.B.; Zhu, J.; Gao, J.F.; Wang, C.; Li, H.; Li, H.; Zhang, H.Q.; Zhang, S.; Wang, D.M.; Wang, Q.X.; et al. Transcription factor AtMYB103 is required for anther development by regulating tapetum development, callose dissolution and exine formation in Arabidopsis. Plant J. 2007, 52, 528-538. [CrossRef] [PubMed]

24. Phan, A.H.; Iacuone, S.; Li, S.F.; Parish, R.W. The MYB80 transcription factor is required for pollen development and the regulation of tapetal programmed cell death in Arabidopsis thaliana. Plant Cell 2011, 23, 2209-2224. [CrossRef] [PubMed]

25. Guo, J.J.; Wang, P.; Cheng, Q.; Sun, L.M.; Wang, H.Y.; Wang, Y.T.; Kao, L.N.; Li, Y.N.; Qiu, T.Y.; Yang, W.C.; et al. Proteomic analysis reveals strong mitochondrial involvement in cytoplasmic male sterility of pepper (Capsicum annuum L.). J. Proteom. 2017, 168, 15-27. [CrossRef] [PubMed]

26. Coller, H.A.; Grandori, C.; Tamayo, P.; Colbert, T.; Lander, E.S.; Eisenman, R.N.; Golub, T.R. Expression analysis with oligonucleotide microarrays reveals that MYC regulates genes involved in growth, cell cycle, signaling, and adhesion. Proc. Natl. Acad. Sci. USA 2000, 97, 3260-3265. [CrossRef] [PubMed]

27. Nesbit, C.E.; Grove, L.E.; Yin, X.; Prochownik, E.V. Differential apoptotic behaviors of c-Myc, N-Myc, and L-myc oncoproteins. Cell Growth Differ. 1998, 9, 731-741. [PubMed]

28. Barrett, J.; Birrer, M.J.; Kato, G.J.; Dosaka-Akita, H.; Dang, C.V. Activation domains of L-Myc and c-Myc determine their transforming potencies in rat embryo cells. Mol. Cell. Biol. 1992, 12, 3130-3137. [CrossRef] [PubMed]

29. Blackwood, E.M.; Eisenman, R.N. Max: A helix-loophelix zipper protein that forms a sequence-specific DNA-binding complex with myc. Science 1991, 251, 1211-1217. [CrossRef] [PubMed]

30. Ferredamare, A.R.; Pognonec, P.; Roeder, R.G.; Burley, S.K. Structure and function of the b/HLH/Z domain of USF. EMBO J. 1994, 13, 180-189.

31. Ellenberger, T.; Fass, D.; Arnaud, M.; Harrison, S.C. Crystal structure of transcription factor E47: E-Box recognition by a basic region helix-loop-helix dimer. Genes Dev. 1994, 8, 970-980. [CrossRef] [PubMed] 
32. Henriksson, M.; Luscher, B. Proteins of the Myc network: Essential regulators of cell growth and differentiation. Adv. Cancer Res. 1996, 68, 109-182. [PubMed]

33. Ledent, V.; Vervoort, M. The basic helix-loop-helix protein family: Comparative genomics and phylogenetic analysis. Genome Res. 2001, 11, 754-770. [CrossRef] [PubMed]

34. Carretero-Paulet, L.; Galstyan, A.; Roig-Villanova, I.; Martínez-García, J.F.; Bilbao-Castro, J.R.; Robertson, D.L. Genome-wide classification and evolutionary analysis of the bHLH family of transcription factors in Arabidopsis, poplar, rice, moss, and algae. Plant Physiol. 2010, 153, 398-1412. [CrossRef] [PubMed]

35. Goodrich, J.; Carpenter, R.; Coen, E.S. A common gene regulates pigmentation pattern in diverse plant species. Cell 1992, 68, 955-964. [CrossRef]

36. Rajani, S.; Sundaresan, V. The Arabidopsis myc/bHLH gene ALCATRAZ enables cell separation in fruit dehiscence. Curr. Biol. 2001, 11, 1914-1922. [CrossRef]

37. Breitbart, R.E.; Andreadis, A.; Nadal-Ginard, B. Alternative splicing: A ubiquitous mechanism for the generation of multiple protein isoforms from single genes. Annu. Rev. Biochem. 1987, 56, 467-495. [CrossRef] [PubMed]

38. Nilsen, T.W.; Graveley, B.R. Expansion of the eukaryotic proteome by alternative splicing. Nature 2010, 463, 457-463. [CrossRef] [PubMed]

39. Forrest, S.T.; Barringhaus, K.G.; Perlegas, D.; Hammarskjold, M.L.; McNamara, C.A. Intron retention generates a novel Id3 isoform that inhibits vascular lesion formation. J. Biol. Chem. 2004, 279, 32897-32903. [CrossRef] [PubMed]

40. Kadesch, T. Consequences of heteromeric interactions among helix-loop-helix proteins. Cell Growth Differ. 1993, 4, 49-55. [PubMed]

41. Massari, M.E.; Murre, C. Helix-loop-helix proteins: Regulators of transcription in eucaryotic organisms. Mol. Cell. Biol. 2000, 20, 429-440. [CrossRef] [PubMed]

42. Ariizumi, T.; Toriyama, K. Pollen exine pattern formation is dependent on three major developmental processes in Arabidopsis thaliana. Int. J. Plant Dev. Biol. 2007, 1, 106-115.

43. Raghavan, V. Pollen abortion and male sterility. In Molecular Embryology of Flowering Plants, 2nd ed.; Raghavan, V., Ed.; Cambridge University Press: Cambridge, UK, 1997; pp. 120-151, ISBN 9780511574528.

44. Liu, L.; Fan, X.D. Tapetum: Regulation and role in sporopollenin biosynthesis in Arabidopsis. Plant Mol. Biol. 2013, 83, 165-175. [CrossRef] [PubMed]

45. Xu, J.; Yang, C.Y.; Yuan, Z.; Zhang, D.S.; Gondwe, M.Y.; Ding, Z.W.; Liang, W.Q.; Zhang, D.B.; Wilson, Z.A. The ABORTED MICROSPORES regulatory network is required for postmeiotic male reproductive development in Arabidopsis thaliana. Plant Cell 2010, 22, 91-107. [CrossRef] [PubMed]

46. De Azevedo Souza, C.; Kim, S.S.; Koch, S.; Kienow, L.; Schneider, K.; McKim, S.M.; Haughn, G.W.; Kombrink, E.; Douglas, C.J. A novel fatty Acyl-CoA synthetase is required for pollen development and sporopollenin biosynthesis in Arabidopsis. Plant Cell 2009, 21, 507-525. [CrossRef] [PubMed]

47. Dobritsa, A.A.; Lei, Z.; Nishikawa, S.; Urbanczyk-Wochniak, E.; Huhman, D.V.; Preuss, D. LAP5 and LAP6 encode anther-specific proteins with similarity to chalcone synthase essential for pollen exine development in Arabidopsis. Plant Physiol. 2010, 153, 937-955. [CrossRef] [PubMed]

48. Jiang, J.; Zhang, Z.; Cao, J. Pollen wall development: The associated enzymes and metabolic pathways. Plant Biol. 2013, 15, 249-263. [CrossRef] [PubMed]

49. Wang, H.Y.; Sui, X.L.; Guo, J.J.; Wang, Z.Y.; Cheng, J.T.; Ma, S.; Li, X.; Zhang, Z.X. Antisense suppression of cucumber (Cucumis sativus L.) sucrose synthase 3 (CsSUS3) reduces hypoxic stress tolerance. Plant Cell Environ. 2014, 37, 795-810. [CrossRef] [PubMed]

50. Tang, L.K.; Chu, H.; Yip, W.K.; Yeung, E.C.; Lo, C. An anther-specific dihydroflavonol 4-reductase-like gene (DRL1) is essential for male fertility in Arabidopsis. New Phytol. 2009, 181, 576-587. [CrossRef] [PubMed]

51. Dobritsa, A.A.; Nishikawa, S.; Preuss, D.; Urbanczyk-Wochniak, E.; Sumner, L.W.; Hammond, A.; Carlson, A.L.; Swanson, R.J. LAP3, a novel plant protein required for pollen development, is essential for proper exine formation. Sex. Plant Reprod. 2009, 22, 167-177. [CrossRef] [PubMed]

52. Wang, S.B.; Liu, K.W.; Diao, W.P.; Zhi, L.; Ge, W.; Liu, J.B.; Pan, B.G.; Wan, H.J.; Chen, J.F. Evaluation of appropriate reference genes for gene expression studies in pepper by quantitative real-time PCR. Mol. Breed. 2012, 30, 1393-1400. [CrossRef] 
53. Kouchi, H.; Hata, S. Isolation and characterization of novel nodulin cDNAs representing genes expressed at early stages of soybean nodule development. Mol. Gen. Genet. 1993, 238, 106-119. [PubMed] (CC BY) license (http://creativecommons.org/licenses/by/4.0/). 